\title{
Deep Sequencing of Recombinant Virus Populations in Transgenic and Nontransgenic Plants Infected with Cucumber mosaic virus
}

\author{
Marco Morroni, ${ }^{1}$ Mireille Jacquemond, ${ }^{2}$ and Mark Tepfer ${ }^{1,2,3}$ \\ ${ }^{1}$ Plant Virology Group, ICGEB Biosafety Outstation, Ca' Tron di Roncade, I-31056, Italy; ${ }^{2}$ INRA UR407 Pathologie Végétale, \\ 84143 Montfavet, France; ${ }^{3}$ INRA UMR1318 Inst. J.-P. Bourgin, 78026 Versailles cedex, France
}

Submitted 25 February 2013. Accepted 15 March 2013.

\begin{abstract}
Recombination is a major source of virus variability, and the question of whether novel recombinant viruses would emerge in transgenic plants expressing viral sequences has been a biosafety issue. We describe the results of pyrosequencing the recombinant viral RNAs appearing in transgenic plants expressing the coat protein $(\mathrm{CP})$ gene and $3^{\prime}$ noncoding region of Cucumber mosaic virus RNA3, as well as in nontransgenic controls. The populations of recombinants in both transgenic and nontransgenic plants were similar to those previously described from Sanger sequencing but many more recombinant types were observed, including a novel class of large deletions removing all or nearly the entire $\mathrm{CP}$ gene. These results show that populations of recombinant viral genomes arising de novo can be characterized in detail by pyrosequencing, and confirm that the transgenic plants did not harbor novel recombinants of biosafety concern.
\end{abstract}

Members of the family Bromoviridae (tripartite, positivesense RNA viruses that infect higher plants), including Brome mosaic virus, Cucumber mosaic virus (CMV), and its close relative, Tomato aspermy virus (TAV), have become valuable tools for studying recombination in viral genomes. CMV isolates are highly diverse and are classed in three distinct subgroups that have high sequence identity within each subgroup; whereas subgroups IA and IB differ by approximately 8 to $10 \%$, IA and IB differ from subgroup II by approximately 22 to $27 \%$, and CMV and TAV differ by approximately 28 to $37 \%$. In addition to the three genomic RNAs, all cucumoviruses have two subgenomic RNAs: RNA4a corresponds to the 3 '-terminal part of RNA2 and is translated to produce protein $2 \mathrm{~b}$, and RNA4 corresponds to the 3 '-terminal part of RNA3 and is translated to produce the coat protein (CP). CMV subgroup II and TAV strains have an additional RNA, RNA5, corresponding to the $3^{\prime}$-noncoding region $\left(3^{\prime}\right.$-ncr) of the genomic RNAs, with RNAs 2 and 3 contributing the majority of the molecules to the population of RNA5. All cucumoviral RNAs except RNA5 are capped at the $5^{\prime}$ end. Reviews specifically on cucumoviruses and CMV have been published by Palukaitis

Current address for M. Morroni: Bioteck S.p.A., via E. Fermi 49, 36057 Arcugnano, Vicenza, Italy.

Corresponding author: M. Tepfer; E-mail: Mark.Tepfer@versailles.inra.fr; Telephone: +33 (0)130833039.

(C) 2013 The American Phytopathological Society and García-Arenal (2003) and Jacquemond (2012), respectively.

Co-inoculation with highly divergent cloned CMV isolates, or with CMV and TAV, has been used to map and characterize several types of de novo recombination events. In a study on plants co-infected with CMV and TAV, it was first shown that recombinant cucumoviral RNAs could be cloned and sequenced after reverse-transcription polymerase chain reaction (RT-PCR) amplification, even when they represent a minor component of viral RNAs (Aaziz and Tepfer 1999). The same strategy was then used by de Wispelaere and associates (2005) to characterize the populations of recombinants across nearly all of RNA3 in plants co-infected with the two viruses. That study showed a remarkable assortment of recombinant types, including precise homologous recombination at numerous sites across RNA3, imprecise homologous recombination within GU repeat motifs in the $5^{\prime}$-ncr, and nonhomologous recombination in the $3^{\prime}$-ncr, creating molecules structurally similar to certain naturally occurring recombinant isolates of CMV and TAV (Chen et al. 2002; Moreno et al. 1997; Shi et al. 1997). In this class of recombinants, the major parent contributes a nearly complete viral RNA, lacking only the $3^{\prime}$-terminal part of the $3^{\prime}$-ncr, and the minor parent contributes a complete or nearly complete $3^{\prime}$-ncr. We will refer to this class of molecules as $3^{\prime}$-ncr insertion recombinants.

Although recombination is clearly important in the context of virus evolution and host adaptation, since the early 1990s recombination has also been an issue regarding the potential impact of virus-resistant transgenic plants (Thompson and Tepfer 2010). The question, long unanswered, was whether recombination between viral sequences in a transgene mRNA and the RNA of an infecting virus could generate novel viral genomes. A first answer was provided by Turturo and associates (2008), who, using an improved version of the strategy used by de Wispelaere and associates (2005), were able to compare the population of recombinant viruses in nontransgenic plants infected with a subgroup IA and a subgroup II CMV strain (I17F and R, respectively), with the population in transgenic plants expressing an R-CMV coat protein mRNA infected with I17F-CMV. In these experiments using wild-type viruses under minimal selection pressure in favor of recombinants, they observed equivalent populations of recombinants in transgenic and nontransgenic plants. Nonetheless, the conclusions were limited by three factors: i) only 10 recombinants per plant were sequenced; ii) there was an extremely potent hotspot for recombination that completely dominated the results, particularly in the transgenic plants; and iii) the nonhomologous 3 'ncr insertion recombinants that had been observed by de Wispelaere and associates (2005) were not observed, which 
the authors suggested may have been masked by the dominant hotspot. Because the hotspot recombinant is not viable when inoculated alone on plants (Pierrugues et al. 2007), this made it possible to minimize its presence in the recombinant population by exerting selection pressure in favor of recombinants (Morroni et al. 2009). When the same line of R-CMV transgenic plants was inoculated with the slightly disabled I17FdelCMV in order to exert selection pressure in favor of recombinants, as expected, the hotspot recombinant was nearly absent, and numerous types of 3 '-ncr insertion recombinants were observed (Morroni et al. 2009).

The recent increases in the length of pyrosequencing reads made it possible to reexamine, in this study, the samples studied by Turturo and associates (2008) and Morroni and associates (2009), in order to clarify several points. i) Are there differences in the results obtained with Sanger sequencing versus pyrosequencing that would suggest biases? ii) If new types of recombinants are observed, does this provide information regarding the different mechanisms by which recombination occurs? iii) With hundreds or thousands of sequence reads (pyrosequencing) rather than tens (Sanger), are there any recombinants present in transgenic plants and absent in nontransgenics that would suggest potential risks associated with transgenic plants expressing viral sequences? To the best of our knowledge, this is the first report of using pyrosequencing to characterize populations of recombinant viral genomes created de novo under experimental conditions.

\section{RESULTS}

Pyrosequencing I17F- and R-CMV RNA3 recombinants.

Six samples representative of the diversity of those studied by Turturo and associates (2008) and Morroni and associates (2009) were chosen for deep sequencing of recombinants: wild-type plants 5 and 31 infected with both I17F-CMV and R-CMV, transgenic plants 8 and 86 infected with I17F-CMV (Turturo et al. 2008), and samples 10AI33 and 10AM41 (transgenic plants AI33 and AM41 10 days postinoculation [dpi]) infected with the attenuated I17Fdel-CMV (Morroni et al. 2009). In order to specifically amplify recombinant molecules, we used a reverse primer specific to the $3^{\prime}$ end of R-CMV RNA3 and a forward primer specific to a site upstream of the CP gene of I17F-CMV RNA3. These genome segments will further be referred to as $\mathrm{R}_{3}$ and $\mathrm{I}_{3}$, respectively. The RT-PCR products represented a population of molecules of approximately 1,000 to 1,200 nucleotides (nt), formed of $\mathrm{I}_{3}$ sequence at the $5^{\prime}$ end and $\mathrm{R}_{3}$ sequence at the $3^{\prime}$ end, covering nearly all of the $\mathrm{R}_{3}$ sequence expressed in mRNA in the transgenic plants. Because the Titanium Pyrosequencing Kit used in this study can produce reads of up to approximately $600 \mathrm{nt}$, the initial RT-PCR products were reamplified by PCR to generate two contiguous fragment of 500 to $600 \mathrm{nt}$, and pyrosequencing was performed in both directions on these fragments.

\section{Sequence data analysis.}

For reasons that were unexplained by the sequencing provider, there was great heterogeneity in the number of reads per sample and direction of sequencing, which ranged from 0 to more than 29,000 (Table 1) and, overall, plants 86, 10AI33, and 10AM41 were notably under-represented compared with the others. Thus, the analysis of recombinants is essentially qualitative, and the relative abundance of recombinants in the $5^{\prime}$ and $3^{\prime}$ halves cannot be compared with precision. However, on the positive side, non-CMV sequences only represented approximately $0.1 \%$ of the reads. The pyrosequencing reads longer than $100 \mathrm{nt}$ were assembled into contigs, the contig consensus sequences were compared with the wild-type sequences to identify the crossover sites, and, for each contig, the number of

Table 1. Overview of pyrosequencing results ${ }^{\mathrm{a}}$

\begin{tabular}{|c|c|c|c|c|c|c|c|}
\hline Plant name & $\begin{array}{c}\text { Plant line/ } \\
\text { CMV genotype }\end{array}$ & $\begin{array}{c}\text { Region } \\
\text { sequenced }\end{array}$ & $\begin{array}{c}\text { CMV reads } \\
>100 \mathrm{nt}\end{array}$ & $\begin{array}{l}\text { Reads of CMV } \\
\text { recombinants }\end{array}$ & $\begin{array}{c}\text { Reads of } \mathbf{I}_{3} / \mathbf{R}_{3} \\
\text { homologous } \\
\text { recombinants }\end{array}$ & $\begin{array}{c}\text { Reads of } \mathbf{I}_{3} / \mathbf{R}_{\mathbf{3}} \\
\text { nonhomologous } \\
\text { recombinants }\end{array}$ & $\begin{array}{c}\text { Reads of } I_{3} / R_{1} \\
\text { and } I_{3} / R_{2} \\
\text { recombinants }\end{array}$ \\
\hline \multirow[t]{2}{*}{5} & \multirow[t]{2}{*}{ XHFD8/I17F+R } & $\begin{array}{l}5^{\prime} \text { forward } \\
5^{\prime} \text { reverse }\end{array}$ & $\begin{array}{r}8,696 \\
14,525\end{array}$ & $\begin{array}{l}2 \\
5\end{array}$ & 5 & 2 & 0 \\
\hline & & $\begin{array}{l}3^{\prime} \text { forward } \\
3^{\prime} \text { reverse }\end{array}$ & $\begin{array}{l}5,676 \\
2,352\end{array}$ & $\begin{array}{l}49 \\
99\end{array}$ & 32 & 25 & 91 \\
\hline \multirow[t]{2}{*}{31} & \multirow[t]{2}{*}{ XHFD8/I17F+R } & $\begin{array}{l}5^{\prime} \text { forward } \\
5^{\prime} \text { reverse }\end{array}$ & $\begin{array}{r}10,125 \\
7,564 \\
\end{array}$ & $\begin{array}{l}987 \\
877\end{array}$ & 1,856 & 8 & 0 \\
\hline & & $\begin{array}{l}3^{\prime} \text { forward } \\
3^{\prime} \text { reverse }\end{array}$ & $\begin{array}{r}0 \\
2,693 \\
\end{array}$ & $\begin{array}{r}0 \\
156 \\
\end{array}$ & 4 & 22 & 130 \\
\hline \multirow[t]{2}{*}{8} & \multirow[t]{2}{*}{$40.1 / \mathrm{I} 17 \mathrm{~F}$} & $\begin{array}{l}5^{\prime} \text { forward } \\
5^{\prime} \text { reverse }\end{array}$ & $\begin{array}{l}382 \\
210 \\
\end{array}$ & $\begin{array}{l}158 \\
118 \\
\end{array}$ & 276 & 0 & 0 \\
\hline & & $\begin{array}{l}3^{\prime} \text { forward } \\
3^{\prime} \text { reverse }\end{array}$ & $\begin{array}{r}29,209 \\
1,002\end{array}$ & $\begin{array}{l}1 \\
1\end{array}$ & 1 & 1 & 0 \\
\hline \multirow[t]{2}{*}{86} & \multirow[t]{2}{*}{$40.1 / \mathrm{I} 17 \mathrm{~F}$} & $\begin{array}{l}5^{\prime} \text { forward } \\
5^{\prime} \text { reverse }\end{array}$ & $\begin{array}{l}3,560 \\
1,668 \\
\end{array}$ & $\begin{array}{r}335 \\
1,481 \\
\end{array}$ & 1,815 & 1 & 0 \\
\hline & & $\begin{array}{l}3^{\prime} \text { forward } \\
3^{\prime} \text { reverse }\end{array}$ & $\begin{array}{l}292 \\
459\end{array}$ & $\begin{array}{l}0 \\
1\end{array}$ & 0 & 1 & 0 \\
\hline \multirow[t]{2}{*}{ 10AI33 } & \multirow[t]{2}{*}{ 40.1/I17Fdel } & $\begin{array}{l}5^{\prime} \text { forward } \\
5^{\prime} \text { reverse }\end{array}$ & $\begin{array}{r}568 \\
1,507\end{array}$ & $\begin{array}{l}1 \\
0\end{array}$ & 1 & 0 & 0 \\
\hline & & $\begin{array}{l}3^{\prime} \text { forward } \\
3^{\prime} \text { reverse }\end{array}$ & $\begin{array}{l}1,907 \\
2,445\end{array}$ & $\begin{array}{r}224 \\
1,660\end{array}$ & 20 & 1,864 & 0 \\
\hline 10AM41 & 40.1/I17Fdel & $\begin{array}{l}5^{\prime} \text { forward } \\
5^{\prime} \text { reverse }\end{array}$ & $\begin{array}{l}329 \\
945\end{array}$ & $\begin{array}{l}0 \\
0\end{array}$ & 0 & 0 & 0 \\
\hline & & $\begin{array}{l}3^{\prime} \text { forward } \\
3^{\prime} \text { reverse }\end{array}$ & $\begin{array}{r}146 \\
2,246\end{array}$ & $\begin{array}{r}113 \\
2,011\end{array}$ & 0 & 2,124 & 0 \\
\hline Totals & $\ldots$ & $\ldots$ & 98,506 & 8,279 & 4,010 & 4,048 & 221 \\
\hline
\end{tabular}

${ }^{\mathrm{a}} \mathrm{CMV}=$ Cucumber mosaic virus and $\mathrm{nt}=$ nucleotides 
reads including the crossover was tabulated (Table 1). Table 1 further breaks these down into homologous and nonhomologous RNA3 recombinants and other types of recombinants. It should be noted that, because the initial amplified population of $I_{3} / R_{3}$ recombinants of approximately 1,000 to $1,200 \mathrm{nt}$ was sequenced in two fragments, for every read with a crossover, there should be a wild-type read corresponding to the other half of the initial molecule of approximately 1,000 to 1,200 nt. In addition, reads that were too short to reach the crossover site in the 1,000- to 1,200-nt fragment will also be wild-type reads.

\section{$\mathbf{I}_{3} / \mathbf{R}_{\mathbf{3}}$ homologous recombinants.}

As expected with the increase in the number of sequences, many more recombination sites were observed by pyrosequencing than had been detected by Sanger sequencing of cloned recombinants (Tables 2 and 3; Fig. 1). This increase was particularly evident for the nontransgenic plants inoculated with I17F- and R-CMV (Table 2; Fig. 1B), because 24 different crossover sites were observed by pyrosequencing, compared with 7 by Sanger sequencing. There was also a clear increase in the number of crossover sites observed in the transgenic plants inoculated with I17F-CMV (Table 3; Fig. 1D), with five sites instead of just one. In spite of the increased numbers of recombinant sequences observed by pyrosequencing, there were only three cases where a crossover site observed by Sanger sequencing was not also observed by pyrosequencing in the same plant: one in plant 5 and two in plant 31 (Table 2).
However, the region concerned in plant 31 was the only one where pyrosequencing failed completely (Table 1), in spite of three attempts; consequently, it is not surprising that certain crossovers in this region were absent. Further, in two of the three cases, the recombinant in question was observed in the other equivalent plant. Overall, the congruence between Sanger and pyrosequencing suggests that the two techniques did not have specific sampling biases.

One of the remarkable features of the homologous $I_{3} / R_{3}$ recombinants described by Turturo and associates (2008) is that only homologous recombinants were observed, with an extremely prominent hotspot in the block of sequence identity between $I_{3}$ and $R_{3}$ at position $R_{3}$ 1523-1554 (crossover sites will be designated in the text by their nucleotide position on R$\mathrm{CMV}$; the corresponding I17F-CMV positions can be found in the tables). In particular, this was the only crossover site observed in transgenic plants infected with I17F-CMV. This pattern of recombination was fully confirmed by pyrosequencing, the only notable difference being that, with deeper sequencing, additional minor crossover sites were also observed.

As mentioned previously, the hotspot recombinant at $\mathrm{R}_{3} 1523$ 1554 was not viable when inoculated alone (Pierrugues et al. 2007), which made it possible to unmask other recombinants when the hotspot recombinant was counterselected (Morroni et al. 2009). However, in that study, nonhomologous recombinants were predominant in the population, with a single homologous crossover observed in the $3^{\prime}$-ncr at position $\mathrm{R}_{3}$ 1998-2041. Homologous recombination at this site was also observed by

Table 2. Comparison of homologous recombination crossover sites detected by Sanger and pyrosequencing in nontransgenic plants 5 and 31 infected with I17F-CMV and R-CMV ${ }^{\mathrm{a}}$

\begin{tabular}{|c|c|c|c|c|c|}
\hline \multirow[b]{2}{*}{$I_{3}$ crossover site } & \multirow[b]{2}{*}{$R_{3}$ crossover site } & \multicolumn{2}{|c|}{ Plant 5 reads } & \multicolumn{2}{|c|}{ Plant 31 reads } \\
\hline & & Sanger & Pyrosequencing & Sanger & Pyrosequencing \\
\hline \multicolumn{6}{|l|}{$\overline{5^{\prime} \text { part }}$} \\
\hline $1258-1270$ & $1229-1241$ & 0 & 0 & 0 & 2 \\
\hline $1401-1409$ & $1372-1380$ & 0 & 0 & 1 & 12 \\
\hline $1415-1430$ & $1386-1401$ & 0 & 0 & 0 & $105^{\mathrm{b}}$ \\
\hline $1438-1443$ & $1409-1414$ & 0 & 1 & 0 & 0 \\
\hline $1452-1454$ & $1423-1425$ & 0 & 0 & 0 & 11 \\
\hline $1459-1463$ & $1430-1434$ & 0 & 1 & 0 & 0 \\
\hline $1521-1528$ & $1492-1499$ & 0 & 0 & 0 & $153^{\mathrm{b}}$ \\
\hline $1552-1583$ & $1523-1554$ & 9 & $3^{\mathrm{b}}$ & 1 & $1,201^{\mathrm{b}}$ \\
\hline $1589-1625$ & $1560-1596$ & 0 & 0 & 0 & $68^{\mathrm{b}}$ \\
\hline $1627-1631$ & $1598-1602$ & 0 & 0 & 0 & $1^{\mathrm{b}}$ \\
\hline $1660-1664$ & $1631-1635$ & 0 & 0 & 0 & 297 \\
\hline $1666-1685$ & $1637-1656$ & 0 & $2^{\mathrm{b}}$ & 1 & $14^{\mathrm{b}}$ \\
\hline $1709-1724$ & $1680-1695^{\mathrm{c}}$ & 1 & 0 & 0 & $2^{b}$ \\
\hline $1726-1727$ & $1697-1698$ & 0 & 0 & 0 & $1^{\mathrm{b}}$ \\
\hline $1741-1748$ & $1712-1719$ & 0 & 0 & 0 & $1^{\mathrm{b}}$ \\
\hline \multicolumn{6}{|l|}{$3^{\prime}$ part } \\
\hline $1819-1823$ & $1790-1794$ & 0 & 1 & 0 & 0 \\
\hline $1837-1843$ & $1808-1814$ & 0 & 1 & 0 & 0 \\
\hline $1855-1865$ & $1826-1836$ & 0 & 3 & 2 & 0 \\
\hline $1879-1883$ & $1850-1854$ & 0 & 1 & 0 & 0 \\
\hline $1957-1966$ & $1939-1948$ & 0 & 1 & 0 & 1 \\
\hline 1968-1970 & $1950-1952$ & 0 & 0 & 1 & 0 \\
\hline $1978-1984$ & 1960-1966 & 0 & 4 & 0 & 0 \\
\hline $2012-2055$ & $1998-2041$ & 0 & 17 & 4 & 3 \\
\hline 2064-2069 & 2049-2054 & 0 & 1 & 0 & 0 \\
\hline $2105-2114$ & 2089-2098 & 0 & 3 & 0 & 0 \\
\hline Total reads ${ }^{\mathrm{e}}$ & $\ldots$ & 10 & $39^{\mathrm{d}}$ & 10 & $1,872^{\mathrm{d}}$ \\
\hline Total crossovers ${ }^{\mathrm{f}}$ & $\ldots$ & 2 & 13 & 6 & 15 \\
\hline $\begin{array}{l}\text { a For each homolo } \\
\text { site are indicated } \\
\text { and } 3^{\prime} \text { parts were } \\
\text { b Including crosso } \\
\text { b Imprecise homol } \\
{ }^{\mathrm{c}} \text { Im } \\
{ }^{\mathrm{d}} \text { Counting two cr } \\
{ }^{\mathrm{e}} \text { Total homologou } \\
\mathrm{f}^{\mathrm{C}} \text { Total homologou }\end{array}$ & $\begin{array}{l}\text { binant molecule de } \\
\text { lant, the number of } \\
\text { separately. } \\
\text { ived from double-re } \\
\text { mbinant. } \\
\text { for each double rec } \\
\text { ant reads/plant. } \\
\text { ant crossover sites. }\end{array}$ & $\begin{array}{l}\text { d by Turt } \\
\text { ag the cro } \\
\text { olecules. } \\
\text { kes the nu }\end{array}$ & $\begin{array}{l}\text { ociates (2008) the } \\
\text { identified by Sange }\end{array}$ & $\begin{array}{l}\text { coordinate: } \\
\text { hg and by }\end{array}$ & $\begin{array}{l}\text { combination cross } \\
\text { ncing is shown. Th }\end{array}$ \\
\hline
\end{tabular}

Vol. 26, No. 7, 2013 / 803 
pyrosequencing, with additional recombinant reads: six at position $R_{3} 1939-1948$ in the $3^{\prime}$-ncr and one at position $R_{3} 1560$ 1596 in the CP coding region (Table 3; Fig. 1D).

A noteworthy result was the detection in the $5^{\prime}$ fragment analyzed from wild-type plants infected with both I17F- and $\mathrm{R}-\mathrm{CMV}$ of pyrosequencing reads with two precise homologous crossover sites formed by an $\mathrm{R}_{3}$ region flanked by $\mathrm{I}_{3}$ sequences (Fig. 1C). Because the initial RT-PCR amplification was designed to be specific for $I_{3} / R_{3}$ recombinants, this suggests one of two possibilities: most likely, these reads were initially linked to an $\mathrm{I}_{3} / \mathrm{R}_{3}$ recombinant in the $3^{\prime}$ fragment analyzed and, thus, represent triple recombinants with the structure $I_{3} / R_{3} / I_{3} / R_{3}$; or, otherwise, they represent a low level of amplification of $\mathrm{I}_{3}$ from the $3^{\prime}$ primer used in the initial RT-PCR, in which case they would be double recombinants. However, because no nonrecombinant $\mathrm{I}_{3}$ reads were obtained in the $3^{\prime}$ half, the second hypothesis is much less plausible. Of the $14 I_{3} / R_{3} / I_{3}$ reads, 11 , including reads from both plants, involved the hotspot crossover site at position $\mathrm{R}_{3}$ 1523-1554. Overall, the prevalence of crossover sites in these reads was compatible with the pattern observed with the single recombinants, suggesting that the multiple recombinants were generated by the same mechanism as the simple homologous recombinants.

\section{$\mathbf{I}_{3} / \mathbf{R}_{\mathbf{3}}$ nonhomologous recombinants.}

One of the aims of this study was to determine whether the transgenic and nontransgenic plants infected with wild-type CMV (Turturo et al. 2008) harbored nonhomologous insertion recombinants in the $3^{\prime}$-ncr like those described in plants infected with I17Fdel-CMV (Morroni et al. 2009), but which would have been masked by the hotspot in the previous study (Turturo et al. 2008). The nonhomologous $I_{3} / R_{3}$ recombinants identified in plants 5 and 31 (wild-type plants infected with both I17F- and R-CMV) and in plants 8 and 86 (transgenic plants infected with I17F-CMV) are shown in Table 4. These recombinants represent a very small proportion of recombinant pyrosequencing reads from these plants, a total of 60 nonhomologous recombinants reads versus 3,989 reads of homologous recombinants $(1.5 \%)$ (Table 1$)$. As was the case for homologous recombinants, the nontransgenic plants harbored a larger number of different recombinant types than the transgenic plants (14 versus 3) and also more recombinant reads (57 versus 3 ). Surprisingly, 54 of these reads were deletions that removed part or all of the $\mathrm{CP}$ coding region. Although these recombinants are certainly nonviable, detection of this class of recombinants in all four plants gives consistency to their presence in the recombinant population. The six remaining $I_{3} / R_{3}$ nonhomologous recombinant reads were $3^{\prime}$-ncr insertion recombinants, of which the most abundant $\left(I_{3} / R_{3}=2097 / 1900\right)$ was similar, though not identical, to those reported in transgenic plants infected with I17Fdel-CMV (Morroni et al. 2009). It is noteworthy that, in 5 of these 17 nonhomologous $I_{3} / R_{3}$ recombinant types ( 25 reads), including the most abundant insertion and deletion recombinants, the $\mathrm{R}_{3}$ site involved was at or near R-CMV position 1900, which previously had been identified as a hotspot for nonhomologous 3 '-ncr insertion recombination between R-CMV and P-TAV (de Wispelaere et al. 2005) and between I17F- and R-CMV (Morroni et al. 2009).

The nonhomologous $\mathrm{I}_{3} / \mathrm{R}_{3}$ recombinants found in transgenic plants 10AI33 and 10AM41 infected with I17Fdel-CMV are shown in Table 5. They represent the vast majority of recombinants in these plants and, in the most extreme case, plant 10AM41, all 2,124 recombinant reads were of this type (Table $1)$. All the recombinants of the nonhomologous $I_{3} / R_{3}$ type previously detected by Morroni and associates (2009) were confirmed by pyrosequencing, and four new ones were identified (Table 5). As with the others, these recombinants also had a $3^{\prime}$ terminal insertion that replaced the region that had been mutated in $\mathrm{I}_{3}$ in order to exert high selection pressure in favor of recombinants. In the previous study (Morroni et al. 2009), recombinants $3 / 3 \mathrm{a}$ and $3 / 3 \mathrm{~h}$ were found in just one of these two samples, whereas here they have been detected in both, highlighting once more that pyrosequencing does, indeed, provide a more complete view of the populations of recombinants.

\section{Other CMV recombinants.}

A few recombinants derived from recombination between $\mathrm{I}_{3}$ and R-CMV RNA1 $\left(\mathrm{R}_{1}\right)$ and RNA2 $\left(\mathrm{R}_{2}\right)$ were also identified in nontransgenic plants infected with both I17F- and R-CMV. These molecules consisted of four homologous and three nonhomologous recombinants (Table 6 ). Four different $I_{3} / R_{1}$ recombinants were found in plant 5 , while two $I_{3} / R_{2}$ and one $I_{3} / R_{1}$ recombinant were found in plant 31 . Interestingly, there was a certain consistency in the crossovers identified, with the positions $\mathrm{I}_{3}$ 2011-2052, $\mathrm{I}_{3}$ 2097-2098, and $\mathrm{R}_{1} 3083$ implicated in two different recombinant types each (one in plant 5 and one in plant 31). Moreover, five of the seven recombinant types were represented by more than a single read, with the two most abundant having 84 and 112 reads, confirming their real presence into the samples.

Table 3. Comparison of homologous recombinants detected by Sanger and pyrosequencing (Pyroseq.) in transgenic plants 8 and 86 infected by I17F-CMV and in transgenic plants 10AI33 and 10AM41 infected by I17Fdel-CMV ${ }^{\mathrm{a}}$

\begin{tabular}{|c|c|c|c|c|c|c|c|c|c|}
\hline \multirow[b]{3}{*}{$\underline{\mathbf{I}_{3} \text { crossover site }}$} & \multirow[b]{3}{*}{$\mathbf{R}_{3}$ crossover site } & \multicolumn{8}{|c|}{ Reads } \\
\hline & & \multicolumn{2}{|c|}{ Plant 8} & \multicolumn{2}{|c|}{ Plant 86} & \multicolumn{2}{|c|}{ Plant 10AI33 } & \multicolumn{2}{|c|}{ Plant 10AM41 } \\
\hline & & Sanger & Pyroseq. & Sanger & Pyroseq. & Sanger & Pyroseq. & Sanger & Pyroseq. \\
\hline \multicolumn{10}{|l|}{$5^{\prime}$ part } \\
\hline $1552-1583$ & $1523-1554$ & 10 & 276 & 10 & 1,812 & 0 & 0 & 0 & 0 \\
\hline $1589-1625$ & $1560-1596$ & 0 & 0 & 0 & 1 & 0 & 1 & 0 & 0 \\
\hline $1633-1634$ & $1604-1605$ & 0 & 0 & 0 & 1 & 0 & 0 & 0 & 0 \\
\hline $1666-1685$ & $1637-1656$ & 0 & 0 & 0 & 1 & 0 & 0 & 0 & 0 \\
\hline \multicolumn{10}{|l|}{$3^{\prime}$ part } \\
\hline $1837-1843$ & $1808-1814$ & 0 & 1 & 0 & 0 & 0 & 0 & 0 & 0 \\
\hline $1957-1966$ & $1939-1948$ & 0 & 0 & 0 & 0 & 0 & 6 & 0 & 0 \\
\hline 2012-2055 & $1998-2041$ & 0 & 0 & 0 & 0 & 1 & 14 & 0 & 0 \\
\hline Total reads ${ }^{\mathrm{b}}$ & $\ldots$ & 10 & 277 & 10 & 1,815 & 1 & 21 & 0 & 0 \\
\hline Total crossovers ${ }^{\mathrm{c}}$ & $\ldots$ & 1 & 2 & 1 & 4 & 1 & 3 & 0 & 0 \\
\hline
\end{tabular}

${ }^{a}$ For each homologous recombinant molecule detected here and by Turturo and associates (2008) and Morroni and associates (2009), the $\mathrm{I}_{3}$ and $\mathrm{R}_{3}$ coordinates of the recombination crossover site are indicated. For each plant, the number of read containing the crossover site identified by Sanger sequencing and by pyrosequencing is shown. The $5^{\prime}$ and $3^{\prime}$ parts were sequenced separately.

b Total homologous recombinant reads/plant.

c Total homologous recombinant crossover sites. 

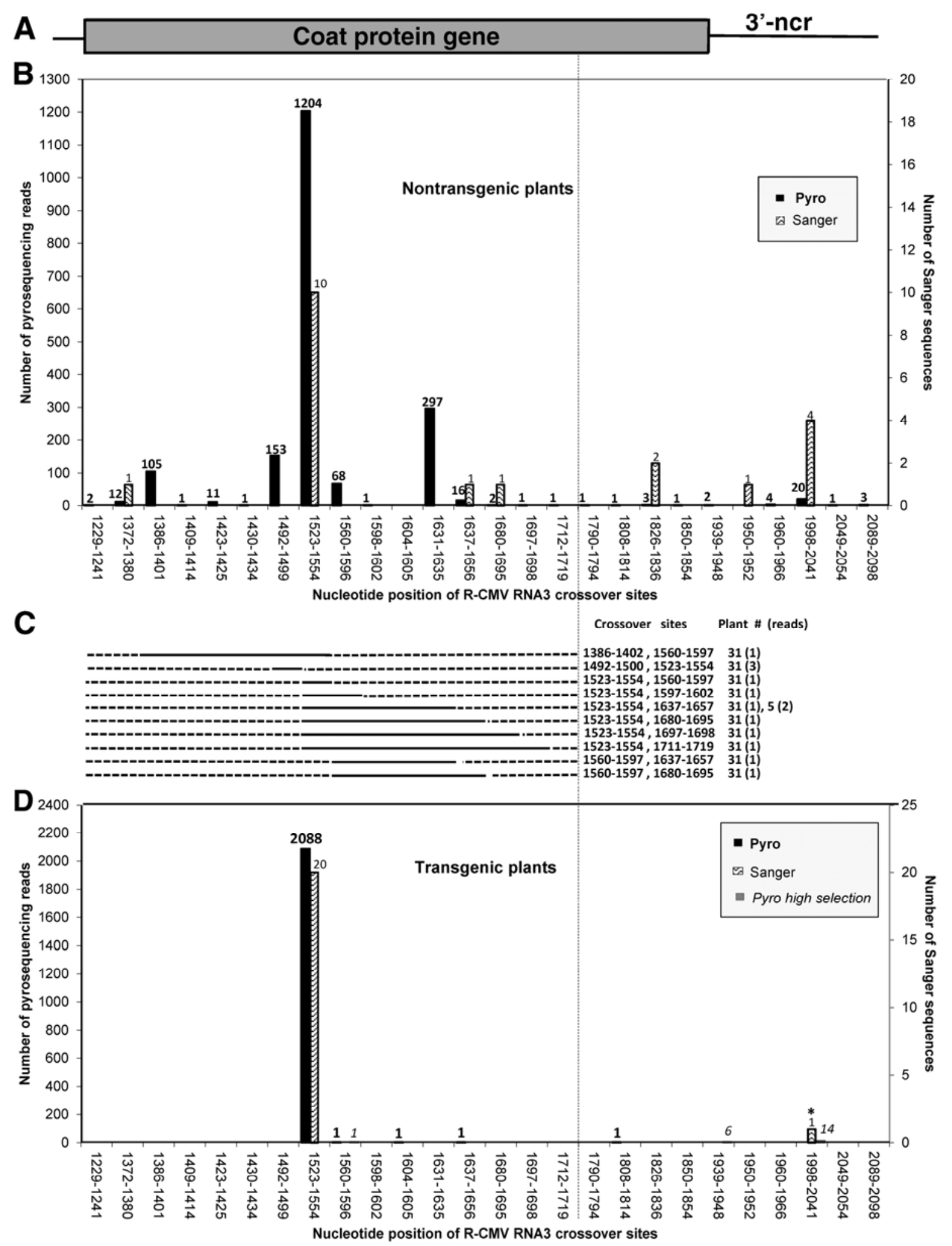

Fig. 1. Map of crossover sites of homologous recombination. A, Schematic diagram of the area of Cucumber mosaic virus (CMV) RNA3 that was studied. The coat protein open reading frame is indicated by a box, the noncoding regions by lines. A vertical dashed line separates the $5^{\prime}$ and $3^{\prime}$ parts that were pyrosequenced separately. B, Position of homologous crossover sites within blocks of sequence identity between R-CMV and I17F-CMV in nontransgenic plants infected with both viruses. The results of Sanger sequencing described by Turturo and associates (2008) are shown in hatched bars, the results of pyrosequencing in solid bars. The number of reads is shown above the bars. C, Double recombinants identified by pyrosequencing of samples from nontransgenic plants infected with both I17F- and R-CMV. I17F- and R-CMV sequences are indicated by dashed and solid lines, respectively. Positions of the crossovers (changes between dashed and solid lines) are those at the corresponding positions in parts B and D, and are indicated to the right of each line, followed by the plant identification number and the number of reads for each double recombinant. D, Position of homologous crossover sites within blocks of sequence identity between transgene mRNA containing R-CMV sequences and I17F-CMV RNA3 in transgenic plants. The results of Sanger sequencing described by Turturo and associates (2008) are shown in hatched bars, except the one at position $\mathrm{R}_{3}$ 1998-2041 (*), which was from conditions of high selection pressure (Morroni et al. 2009). Results of pyrosequencing are shown in solid bars. Results of pyrosequencing from plants of the same transgenic line infected with the defective I17Fdel-CMV are shown in solid gray bars. The number of reads is shown above the bars for pyro, Sanger, and pyro high selection in bold, normal, and italic font, respectively. 


\section{DISCUSSION}

\section{General features of the populations of recombinants.}

One of the questions that motivated this study was whether deep sequencing would provide results that are coherent with those obtained by analysis of cloned recombinants. Although pyrosequencing yielded a more complex vision of the populations of recombinants, overall the picture to emerge was quite compatible with previous results obtained with Sanger sequencing. As expected, the vast majority of recombinants in wild-type plants infected with $\mathrm{I} 17 \mathrm{~F}$ - and R-CMV, as well as in $\mathrm{CP}$ transgenics infected with wild-type I17F-CMV, were of the homologous type. The results confirm the importance of the hotspot in the $\mathrm{CP}$ gene at position $\mathrm{R}_{3}$ 1523-1554; however, many more crossover sites were detected by pyrosequencing than previously (Turturo et al. 2008). Similarly, in the CPtransgenic plants infected with I17Fdel-CMV, as previously described (Morroni et al. 2009), the 3'-ncr insertion recombinants predominated. It is noteworthy that, in the previous study of recombination in nontransgenic plants, the hotspot at position $\mathrm{R}_{3}$ 1523-1554 was not strong in plant 31 (Turturo et al. 2008) whereas, with deeper sequencing, this hotspot was clearly evident in this plant, suggesting that the sampling of 10 clones/plant by Turturo and associates (2008) was insufficient for correct representation of the recombinant population. However, even with the most deeply sequenced samples, with on the order of 1,800 to 2,000 reads, pyrosequencing yielded singlet recombinant reads, which suggests that the complexity of the population has not been fully exhausted. These singlet reads represent genuine recombinants because, in all cases, there were at least four informative nucleotide positions on either side of the crossover.

\section{The diversity of nonhomologous recombinants in wild-type and transgenic plants.}

Nonhomologous recombinants were a minor component of recombinant populations in wild-type plants and, among them, the most abundant were the members of a new class of large deletions that removed all or nearly all of the $\mathrm{CP}$ gene, although we also observed 3 '-ncr insertion recombinants similar but not identical to those described previously in transgenic plants infected with I17Fdel-CMV (Table 5). It had been noted previously in both naturally occurring recombinant isolates (Chen et al. 2002; Moreno et al. 1997; Shi et al. 1997) and recombinants created de novo under experimental conditions (de Wispelaere et al. 2005; Fernandez-Cuartero et al. 1994; Masuta et al. 1998;

Table 4. Structure of nonhomologous $\mathrm{I}_{3} / \mathrm{R}_{3}$ recombinants detected by pyrosequencing in wild-type plants 5 and 31 infected with I17F-CMV and R-CMV $I_{3}$ and in transgenic plants 8 and 86 infected with I17F-CMV



(continued on next page)

\footnotetext{
${ }^{a}$ The position of the last nucleotides of $\mathrm{I}_{3}$ and the first nucleotides of $\mathrm{R}_{3}$ are indicated. Where a stretch of sequence is indicated, this is because recombination occurred within a block of sequence identity between $\mathrm{I}_{3}$ and $\mathrm{R}_{3}$.
} 
Morroni et al. 2009; Pita and Roossinck 2013; Shi et al. 2009; Suzuki et al. 2003) that many of the nonhomologous recombinants resulted from the fusion of the $5^{\prime}$ end of RNA5 (corresponding to $\mathrm{R}_{3}$ position 1899-2001) with other viral sequences. The importance of RNA5 was confirmed in this study because, of the total 27 types of nonhomologous recombinants, nine involved a crossover at the $5^{\prime}$ end of RNA5. This included four types of $I_{3} / R_{3} C P$ deletion recombinants, three types of $I_{3} / R_{3}$ $3^{\prime}$-ncr insertion recombinants, and two types of $I_{3} / R_{1} 3^{\prime}$-ncr insertion recombinants. Considering the high sequence identity shared by the 3'-ncr of CMV RNAs, it was not surprising to detect $I_{3} / R_{2}$ and $I_{3} / R_{1}$ recombinants, both homologous and nonhomologous; however, it was of interest that two nonhomologous $I_{3} / R_{1}$ recombinant types represented by 88 reads involved $R_{1}$ position 3083 , which corresponds exactly to the $R_{3}$ hotspot at position 1900-1902, the 5' end of RNA5.

\section{Mechanisms of recombination.}

The diversity of the populations of both homologous and nonhomologous recombinants imposes constraints when considering the possible mechanisms of recombination. Homologous recombination is thought to occur via a template switch- ing mechanism, in which the synthesis of nascent strand RNA on the donor template is interrupted, and then re-initiated on an acceptor template. Viewed generally, our results and those obtained previously (Aaziz and Tepfer 1999; de Wispelaere et al. 2005; Turturo et al. 2008) showed homologous recombination occurring primarily within large blocks of sequence identity, which is compatible with this mechanism. However, it is remarkable that all but one of the homologous recombinants observed in this study were perfectly precise even under conditions of little or no selection pressure in favor of recombinants and, thus, required extremely precise base pairing between nascent-strand and acceptor-template-strand RNAs. Surprisingly, however, we observed precise homologous recombination in blocks of sequence identity of only two or three nucleotides, which alone would be too short to ensure precise alignment. However, if $\mathrm{G}: \mathrm{U}$ basepairing is taken into account, the blocks of nascent and acceptor strand that can basepair can be considerably longer than the corresponding blocks of sequence identity. For example, the precise homologous crossover in the trinucleotide of sequence identity at position $R_{3} 1423-1425$ is part of a block of potential nascent- or acceptor-strand basepairing of $10 \mathrm{nt}$ if recombination occurred during (+)-strand

Table 4. (continued from preceding page)




synthesis, or of $6 \mathrm{nt}$ if it occurred during (-)-strand synthesis (Fig. 2). However, because potential nascent- or acceptorstrand G:U basepairing does not flank the three other homologous recombination sites of 2 to 3 nt observed in this study, the mechanism of precise homologous recombination must be compatible with either remarkably short stretches of basepairing between the nascent and acceptor template strands or basepairing occurring over longer segments of sequence complementarity, while tolerating a certain degree of mismatch.

In six of the 27 nonhomologous recombinant types described in Tables 4 to 6 , the junction linking distant sites in the genome was within a block of sequence identity which, in some cases, was long enough (e.g., 6 or $10 \mathrm{nt}$ ) for recombination to have occurred by the same mechanism as for homologous recombinants. For the remaining nonhomologous recombinants, other mechanisms must be considered. Nine recombinant types resulted from the fusion of the $5^{\prime}$ end of RNA5 to other sequences, suggesting an RNA5-specific mechanism. It was previously believed that RNA5 was synthesized by internal initiation on (-)-strand CMV genomic RNAs (Blanchard et al. 1996) and, thus, it was logical to envisage a mechanism in which nonhomologous recombination would occur also by internal initiation at the putative promoter for (+)-RNA5 synthesis to yield recombinants with 3 '-terminal RNA5 sequences (Suzuki et al. 2003). However, de Wispelaere and Rao (2009) showed more recently that, in fact, RNA5 is produced by cleavage or degradation of the genomic (+)-sense RNAs in the absence of viral replication. They proposed that recombination occurs during (-)-strand synthesis, and that the replicase, upon reaching the $5^{\prime}$ end of a (+)-sense RNA5 template, reinitiates at the 3 -terminal promoter for (-)-sense RNA synthesis, to yield a recombinant with two complete copies of the $3^{\prime}$-ncr. In contrast to their results, we have not observed recombinants with two complete $3^{\prime}$-ncr regions but, instead, recombinants that resulted from the fusion of the $5^{\prime}$ end of RNA5 to a total of nine different sites on I17F-CMV RNA3, within both the 3'ncr and the CP gene. There is no evidence that these sites are promoters, and recombination was not associated with precise RNA structural features in the region of RNA3 where structures have been determined biochemically (Morroni et al. 2009); therefore, this class of recombinants does not correspond to any of the known mechanisms, based on sequence identity, structural

Table 5. Structure of nonhomologous $\mathrm{I}_{3} / \mathrm{R}_{3}$ recombinants detected by Sanger and pyrosequencing in transgenic plants AI33 and AM41 infected with I17FdelCMV

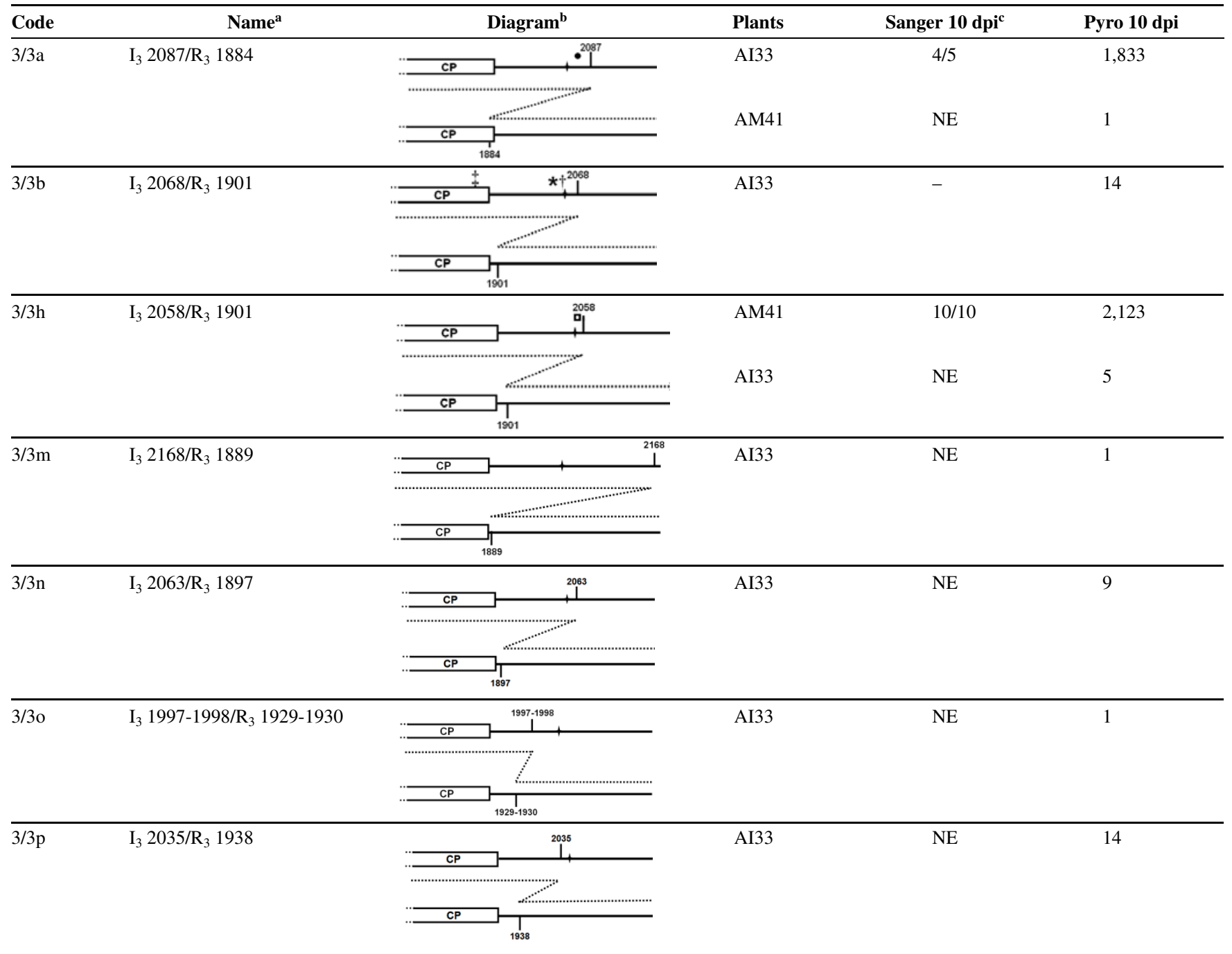

a The position of the last nucleotides of $I_{3}$ and the first nucleotides of $R_{3}$ are indicated. Where a stretch of sequence is indicated, this is because recombination occurred within a block of sequence identity between $\mathrm{I}_{3}$ and $\mathrm{R}_{3}$.

${ }^{\mathrm{b}}$ Point mutations on $\mathrm{I}_{3}$ that appeared in certain recombinants are indicated as: substitutions; $\bullet-\mathrm{C}$ to $\mathrm{U}$ at nt $2064, \ddagger-\mathrm{U}$ to $\mathrm{C}$ at $\mathrm{nt} 1894, *-\mathrm{U}$ to $\mathrm{C}$ at nt 2022, and deletions; $\dagger$ - A at nt 2043, 口 - GG at nucleotides 2,055 to 2,056.

${ }^{c}$ Sanger sequences are given as the number of clones with the recombinant as proportion of the number of clones sequenced; NE $=$ not evaluated (plant without symptoms); - recombinant not observed. 
features, or promoters. How these recombinants are produced and whether recombination occurs during (+)- or (-)-strand synthesis will require further studies with either agroinoculated viral RNAs (Kwon and Rao 2012) or semipurified CMV replicase (Hayes and Buck 1990).

\section{The potential biosafety impact of recombination in transgenic plants on viral diversity.}

The vast majority of virus-resistant transgenic plants, including the squash and papaya lines grown commercially, express viral sequences, most often the CP gene (Prins et al. 2008). A long-standing issue regarding the potential impact of such plants has been whether recombination between the transgene mRNA and the RNA of a related virus could lead to novel recombinant genomes. If so, this could potentially lead to emergence of viruses with modified disease characteristics (Thompson and Tepfer 2010).

From this point of view, it is interesting to consider the viability the recombinant viruses detected by pyrosequencing that had been tested previously when inoculated without wild-type virus (Pierrugues et al. 2007). As was mentioned previously, the hotspot recombinant at $\mathrm{R}_{3}$ 1523-1554 is not viable. Further, among the other crossovers within the $\mathrm{CP}$ gene, recombination at $\mathrm{R}_{3}$ 1680-1695 produced a weakened virus, whereas crossovers at $R_{3}$ 1372-1380, $R_{3}$ 1386-1401, and $R_{3}$ 1560-1596 had normal viability (Pierrugues et al. 2007). Because 3'-ncr insertion recombinants have been described among natural isolates of CMV and TAV (Chen et al. 2002; Moreno et al. 1997; Shi et al. 1997), it is not surprising that $3^{\prime}$-ncr recombinants are viable, as was shown for homologous recombinants at $R_{3} 1939$ 1948 and $R_{3}$ 1998-2041, as well as nonhomologous ones at $I_{3} / R_{3} 2072 / 1899$ and $I_{3} / R_{3}$ 2077/1900 (Pierrugues et al. 2007). Because certain $3^{\prime}$-ncr insertion recombinants (Chen et al. 2002; Fernandez-Cuartero et al. 1994) can have a selective advantage compared with the parental viruses, it is not possible to predict for a given recombinant whether it will be selected either positively or negatively, particularly because this may vary with host species (Chen et al. 2002).

Thus, the critical question is whether recombinants are created in transgenic plants that are not created in nontransgenic ones when infected with two viruses. In spite of a great increase in the number of recombinant types detected by pyrosequencing, it is striking that only one singlet read of homologous recombination was observed at a crossover site (Fig. 1, position $R_{3}$ 1604-1605) in transgenic plants infected with I17F-CMV that was not observed in nontransgenic plants infected with both I17F- and R-CMV. However, there is no reason to believe that this recombinant was produced by a mechanism different from the others and, thus, we would predict that it would have been detected in the nontransgenic plants if

Table 6. Structure of homologous and nonhomologous $I_{3} / R_{1}$ and $I_{3} / R_{2}$ recombinants detected by pyrosequencing in nontransgenic plants 5 and 31 infected with I17F-CMV and R-CMV

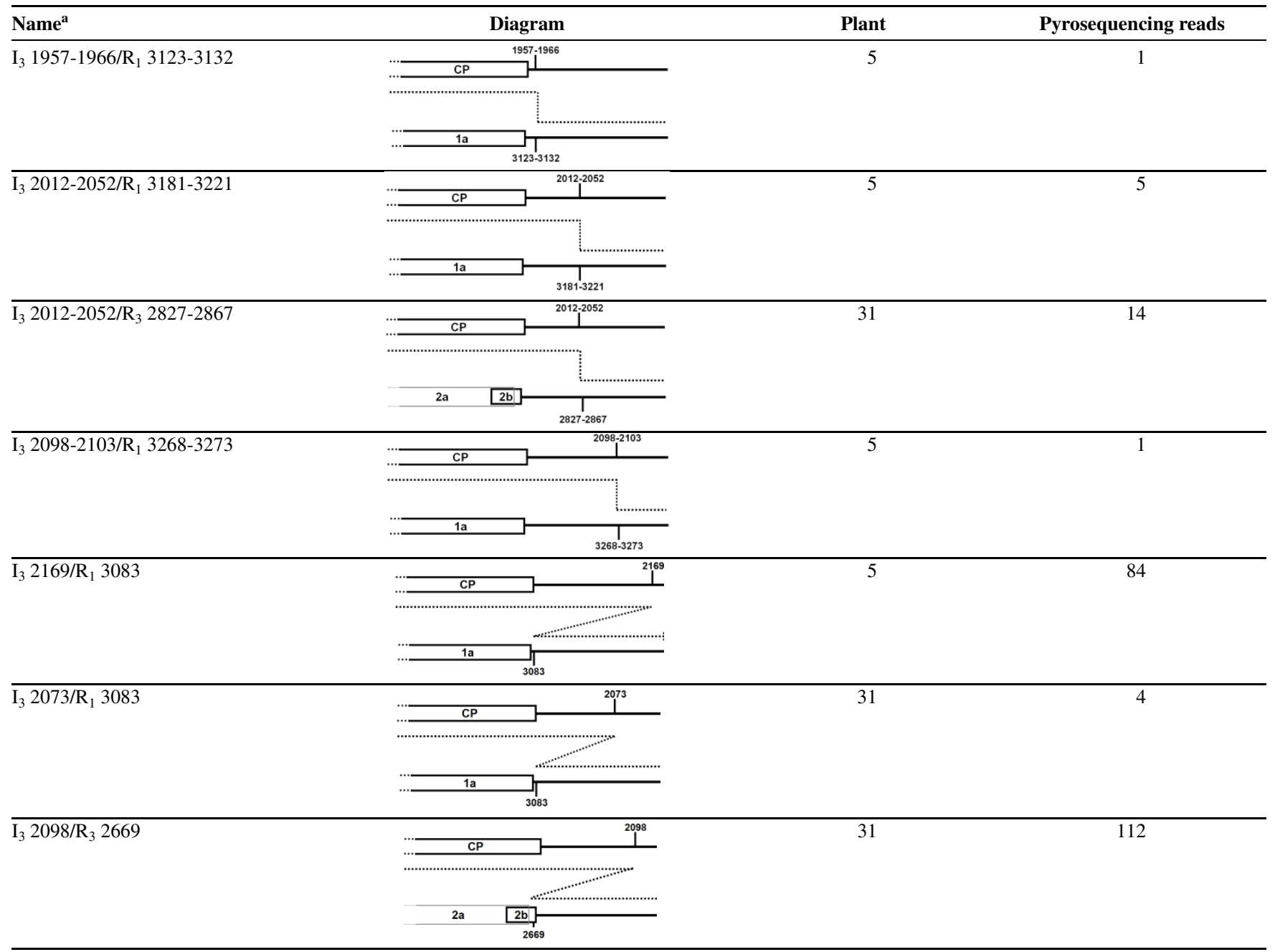

${ }^{a}$ The position of the last nucleotides of $I_{3}$ and the first nucleotides of $R_{1}$ or $R_{2}$ are indicated. Where a stretch of sequence is indicated, this is because recombination occurred within a block of sequence identity. 
more reads had been obtained. Similarly, for nonhomologous recombination, the recombinant types observed in transgenic plants, though not identical to them, are of a type similar to those in the nontransgenic plants. Further, because they involve deletion of nearly the entire $\mathrm{CP}$ gene, they would not be viable if inoculated alone, without wild-type virus.

\section{MATERIALS AND METHODS}

Viruses, plants, and inoculation.

CMV subgroup I strain I17F-CMV (accession numbers HE793683, HE793684, and Y18137) and subgroup II strain RCMV (accession numbers HE793685, HE793686, and Y18138) were maintained as described previously (Turturo et al. 2008). The mutated I17F-CMVdel had a deletion of $6 \mathrm{nt}$ at position 2047-2052 in the 3'-ncr of its RNA3, as described previously (Morroni et al. 2009).

Tobacco plants (Nicotiana tabacum Xanthi XHFD8) were grown in a greenhouse at $25 \pm 2{ }^{\circ} \mathrm{C}$, relative humidity of $75 \pm$ $10 \%$, and day length of $16 \mathrm{~h}$. The transgenic tobacco plants (line 40.5) expressed the CP and the entire $3^{\prime}$-ncr of R-CMV RNA3 (Morroni et al. 2009; Turturo et al. 2008). They exhibited partial resistance to R-CMV but were completely susceptible to I17F-CMV (Turturo et al. 2008) (M. Jacquemond and
M. Tepfer, unpublished). They were inoculated as described previously (Morroni et al. 2009; Turturo et al. 2008).

\section{RNA samples.}

The total RNA samples analyzed were those used in previous studies, as will be described briefly here. Samples of systemically infected leaves were harvested at $7 \mathrm{dpi}$ for co-infected wild-type plants and at $10 \mathrm{dpi}$ for all transgenic plants. The samples were then frozen in liquid nitrogen and stored at $-80^{\circ} \mathrm{C}$ until RNA extraction. Total RNA was extracted from wild-type plants 5 and 31 and from transgenic plants 8 and 86 by Turturo and associates (2008), and from transgenic plants 10AI33 and 10AM41 by Morroni and associates (2009). The extracted RNAs were checked on agarose or on agarose/formaldehyde gels (Morroni et al. 2009; Turturo et al. 2008), quantified by spectrophotometry, and stored at $-80^{\circ} \mathrm{C}$.

\section{RT-PCR.}

First-strand cDNA was synthesized using primer R2191(5'-aacctgtggaaaaccaca-3') with an RNase H- reverse transcriptase to prevent recombination in vitro, as previously described (Turturo et al. 2008), but at 57 instead of $52^{\circ} \mathrm{C}$. PCR was carried out as previously described by the same authors in a final volume of 25 or $50 \mu$ using a reduced concentration of

A Sequence alignment $l_{3}$ and $R_{3}$ surrounding the crossover at $R_{3}$ 1423-1425

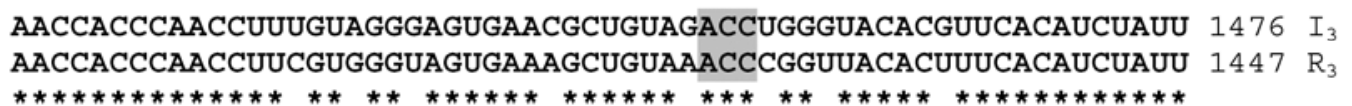

B Potential basepairing during (+)-strand synthesis

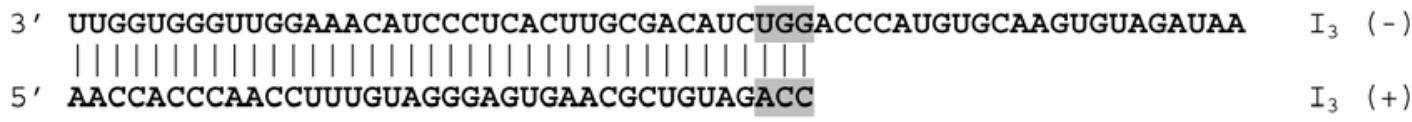

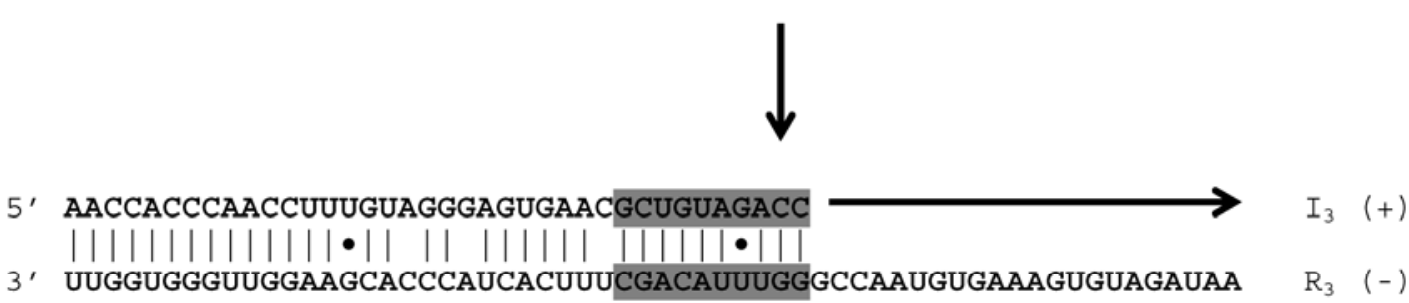

C Potential basepairing during (-)-strand synthesis

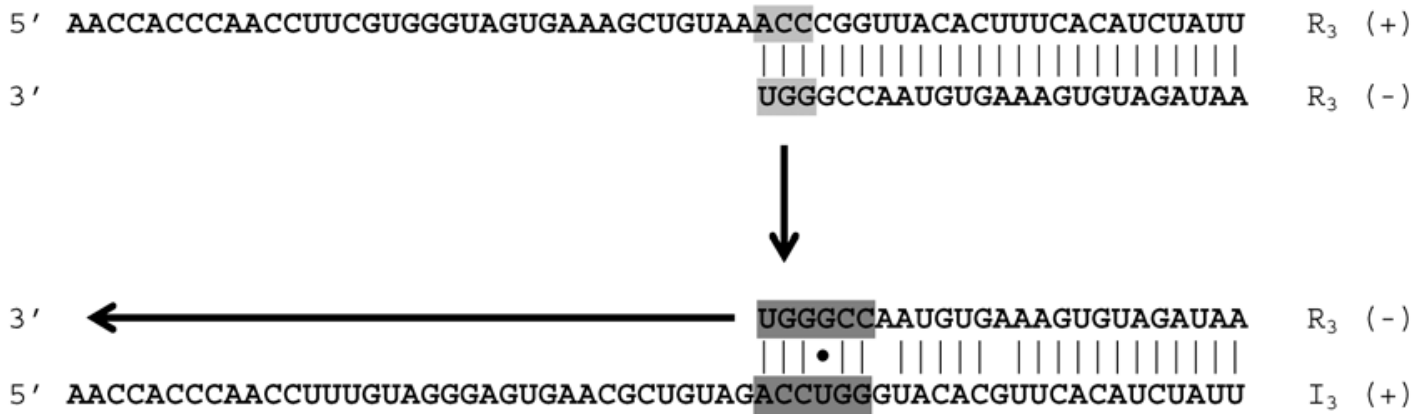

Fig. 2. Potential role of G:U basepairing on determining the sites of precise homologous recombination. The region surrounding the crossover site at the trinucleotide of sequence identity at position $\mathrm{R}_{3}$ 1423-1425 is shown as an example. A, Alignment of $I_{3}$ and $\mathrm{R}_{3}$ surrounding the crossover site (light gray shading); stars indicate identical nucleotides. B and C, Potential basepairing during (+)- and (-)-strand synthesis, respectively, is shaded in dark gray. The vertical arrow indicates the switch of nascent-strand RNA from the donor RNA template (above), and the horizontal arrow indicates reinitiation of nascentstrand RNA synthesis on the acceptor RNA template (below). Basepairing between template and nascent strands is indicated by | for G:C and A:U basepairing and by $\bullet$ for G:U basepairing. 
$\mathrm{MgCl}_{2}$ (1 instead of $\left.1.5 \mathrm{mM}\right)$, generating amplicons of approximately $1.2 \mathrm{~kb}$. Primers were I1188+LNA3 (5'-atTgtCtaCtgact atataga-3', locked nucleic acids shown in upper case), annealing in position 1187-1207 on $\mathrm{I}_{3}$, and primer R2183- (5' -ggaaaa ccacaggcgatac- $3^{\prime}$ ), annealing in $\mathrm{R}_{3}$ position $2165-2183$. The annealing temperature was $60^{\circ} \mathrm{C}$. Negative controls were water (to control for contamination) and a mixture of total RNA extracted from transgenic plants and total RNA extracted from a I17F-CMV-infected nontransgenic tobacco (a control for in vitro recombination). The RT-PCR products were separated on a $1.2 \%$ agarose/Tris-acetate-EDTA gel, purified using the QIAEX II gel extraction kit (Qiagen, Hilden, Germany), and quantified by spectrophotometry.

\section{Pyrosequencing.}

The six RT-PCR amplicons were sent to GATC Biotech, where pyrosequencing was performed on a Roche GS FLX system. Because of the limit of approximately $600 \mathrm{nt}$ of sequence produced with the Titanium Pyrosequencing Kit, the original RT-PCR products (average size $1.2 \mathrm{~kb}$ ) were reamplified by PCR to produce two contiguous segments of 500 to $600 \mathrm{nt}$, corresponding to the $5^{\prime}$ and $3^{\prime}$ halves of the original amplicons, with the pyrosequencing primer and sample-specific tag added to either the $5^{\prime}$ or $3^{\prime}$ end of each half. Thus, for each of the six original RT-PCR amplicons, four different primer pairs were used in order to read both the $5^{\prime}$ and $3^{\prime}$ halves in both forward and reverse direction. For the $5^{\prime}$ half, the primers included a $5^{\prime}$ viral sequence corresponding to $I_{3}$ position $1187-1214$ and a $3^{\prime}$ viral sequence corresponding to a block of sequence identity between I17F- and R-CMV (nucleotide position 1773-1787 of $\mathrm{I}_{3}$ or $1744-1758$ of $\mathrm{R}_{3}$ ). For the $3^{\prime}$-half, the primers included a $5^{\prime}$ viral sequence corresponding to the same block of sequence identity and a $3^{\prime}$ viral sequence corresponding to $R_{3}$ position 2165-2183. The 5'-half and $3^{\prime}$-half PCR products were pyrosequenced in both the forward and reverse sense.

\section{Data analysis.}

In order to segregate the sequence reads into the different recombinant types present, reads $>100 \mathrm{nt}$ were assembled into contigs using default parameters of SeqMan software (DNASTAR Lasergene, Madison, WI, U.S.A.), except that the minimum percentage match was set to $95 \%$. For data sets where this generated more than 10 singleton contigs, these were reanalyzed with minimum match set to $85 \%$. In order to identify the crossover sites, the consensus sequence of each contig was aligned against the six parental viral sequences using the National Center for Biotechnology Information BLASTn with default parameters, except word size was set to 15. Crossover sites were further verified by alignments using the Vector NTI Advance 9 software package (Invitrogen, Carlsbad, CA, U.S.A.).

\section{ACKNOWLEDGMENTS}

We thank A. Friscina for providing the RNA samples from plants 5, 31, 8, and 86. This study was supported by an AIP Bio-Resources from the Institut National de la Recherche Agronomique (INRA) and by the Fondazione Cassamarca.

\section{LITERATURE CITED}

Aaziz, R., and Tepfer, M. 1999. Recombination between genomic RNAs of two cucumoviruses under conditions of minimal selection pressure. Virology 263:282-289.

Blanchard, C. L., Boyce, P. M., and Anderson, B. J. 1996. Cucumber mosaic virus RNA 5 is a mixed population derived from the conserved $3^{\prime}$ terminal regions of genomic RNAs 2 and 3. Virology 217:598-601.

Chen, Y.-K., Goldbach, R., and Prins, M. 2002. Inter- and intramolecular recombinations in the Cucumber mosaic virus genome related to adaptation to Alstroemeria. J. Virol. 76:4119-4124.

de Wispelaere, M., and Rao, A. L. N. 2009. Production of Cucumber mosaic virus RNA5 and its role in RNA recombination. Virology 384:179191.

de Wispelaere, M., Gaubert, S., Trouilloud, S., Belin, C., and Tepfer, M. 2005. A map of the diversity of RNA3 recombinants appearing in plants infected with Cucumber mosaic virus and Tomato aspermy virus. Virology 331:117-127.

Fernández-Cuartero, B., Burgyán, J., Aranda, M. A., Salánki, K., Moriones, E., and García-Arenal, F. 1994. Increase in the relative fitness of a plant virus RNA associated with its recombinant nature. Virology 203:373-377.

Hayes, R. J., and Buck, K. W. 1990. Complete replication of a eukaryotic virus RNA in vitro by a purified RNA-dependent RNA polymerase. Cell 63:363-368.

Jacquemond M. 2012. Cucumber mosaic virus. Adv. Virus Res. 84:439504.

Kwon, S.-J., and Rao, A. L. N. 2012. Emergence of distinct Brome mosaic virus recombinants is determined by the polarity of the inoculum RNA. J. Virol. 86:5204-5220.

Masuta, C., Ueda, S., Suzuki, M., and Uyeda, I. 1998. Evolution of a quadripartite hybrid virus by interspecific exchange and recombination between replicase components of two related tripartite RNA viruses. Proc. Natl. Acad. Sci. U.S.A. 95:10487-10942.

Moreno, I. M., Bernal, J. J., Garcia de Blas, B., Rodriguez-Cerezo, E., and Garcia-Arenal, F. 1997. The expression level of the 3a movement protein determines differences in severity of symptoms between two strains of tomato aspermy cucumovirus. Mol. Plant-Microbe Interact. 10:171-179.

Morroni, M., Thompson, J. R., and Tepfer, M. 2009. Analysis of recombination between viral RNAs and transgene mRNA under conditions of high selection pressure in favour of recombinants. J. Gen. Virol. 90:2798-2807.

Palukaitis, P., and García-Arenal, F. 2003. Cucumoviruses. Adv. Virus Res. 62:242-323.

Pierrugues, O., Guilbaud, L., Fernandez-Delmond, I., Fabre, F., Tepfer, M., and Jacquemond, M. 2007. Biological properties and relative fitness of inter-subgroup Cucumber mosaic virus RNA3 recombinants produced in vitro. J. Gen. Virol. 88:2852-2861.

Pita, J. S., and Roossinck, M. J. 2013. Fixation of emerging interviral recombinants in Cucumber mosaic virus populations. J. Virol. 87:12641269.

Prins, M., Laimler, M., Noris, E., Schubert, J., Wasseneger, M., and Tepfer, M. 2008. Strategies for antiviral resistance in transgenic plants. Mol. Plant Pathol. 9:73-83.

Shi, B.-J., Ding, S. W., and Symons, R. H. 1997. Two novel subgenomic RNAs derived from RNA 3 of tomato aspermy cucumovirus. J. Gen. Virol. 78:505-510.

Shi, B.-J., Symons, R. H., and Palukaitis, P. 2009. Stability and competitiveness of interviral recombinant RNAs derived from a chimeric cucumovirus. Virus Res. 140:216-221.

Suzuki, M., Hibi, T., and Masuta, C. 2003. RNA recombination between cucumoviruses: Possible role of predicted stem-loop structures and an internal subgenomic promoter-like motif. Virology 306:77-86.

Thompson, J. R., and Tepfer, M. 2010. Assessment of the benefits and risks for engineered virus resistance. Adv. Virus Res. 76:33-56.

Turturo, C., Friscina, A., Gaubert, S., Jacquemond, M., Thompson, J. R., and Tepfer, M. 2008. Evaluation of potential risks associated with recombination in transgenic plants expressing viral sequences. J. Gen. Virol. 89:327-335. 